\title{
Effect of Wrinkles on the Surface Area of Graphene: Toward the Design of Nanoelectronics
}

\author{
Zhao Qin, ${ }^{\dagger, \ddagger}$ Michael Taylor, ${ }^{\S, \#}$ Mary Hwang, ${ }^{\dagger, \ddagger}$ Katia Bertoldi, ${ }^{,}$, ,॥ and Markus J. Buehler ${ }^{*}, \dagger, \ddagger$
}

${ }^{\dagger}$ Laboratory for Atomistic and Molecular Mechanics (LAMM), Department of Civil and Environmental Engineering, Massachusetts Institute of Technology, 77 Massachusetts Avenue, Room 1-239, Cambridge, Massachusetts 02139, United States

${ }^{\ddagger}$ Center for Computational Engineering, Massachusetts Institute of Technology, 77 Massachusetts Avenue, Cambridge, Massachusetts 02139, United States

${ }^{\S}$ School of Engineering and Applied Sciences, Harvard University, 29 Oxford Street, Cambridge, Massachusetts 02138, United States

"Kavli Institute, Harvard University, Cambridge, Massachusetts 02138, United States

Supporting Information

ABSTRACT: Graphene has attracted intense attention to the use in extreme applications. However, its small thickness facilitates wrinkle formation, and it is not clear how such structural change affects its area-specific capacitance. Herein, we combine molecular dynamics and continuum mechanics-based simulations to study the changes in surface area induced by wrinkles. We find that the high specific surface area of graphene can only be affected up to $2 \%$ regardless of loading conditions, geometry, and defects.

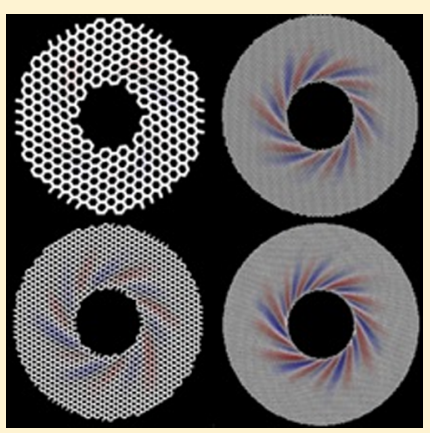

KEYWORDS: Graphene, graphene wrinkling, MD simulations, surface area

$G$ raphene represents an ideal two-dimensional material composed of a single layer of atoms organized in a hexagonal lattice and connected by $\mathrm{sp}^{2}$ in-plane carbon-carbon bonds. $^{1-6}$ It exhibits a high strength $(\sim 118 \mathrm{GPa})$ and elastic modulus $(\sim 1 \mathrm{TPa}),{ }^{7}$ high specific surface area $\left(\sim 2600 \mathrm{~m}^{2} / \mathrm{g}\right),{ }^{8}$ and high electrical and thermal conductivity, making it a perfect candidate as an electrode material in applications that require area-specific capacitance and long life, ${ }^{9,10}$ such as supercapacitors. Furthermore, graphene's ultrathin nature allows it to easily bend, fold, and scroll without rupture, forming continuous wrinkles akin to fingerprint patterns. ${ }^{11-13}$ For this reason, graphene has tremendous potential for use in flexible devices such as foldable displays and stretchable electronics for data storage. $9,14,15$ Nevertheless, these applications involve nonlinear complex deformation, and limits in knowledge about the relationship between wrinkling, defects, and area-specific capacitance significantly impede an evaluation of the robustness of graphene for these applications.

In this Letter, we use a combination of molecular dynamics (MD) and continuum mechanics-based simulations to show that surface area changes induced by wrinkling in loaded freestanding sheets of graphene are small with respect to changes in loading conditions and geometry. Furthermore, we investigate the effect of defects on the changes in surface area and identify a critical threshold below which the wrinkle pattern is minimally altered by the defects. Wrinkling in graphene has been a topic of much research in recent years and has been studied within the context of substrate deposition, ${ }^{16-18}$ ripples in unloaded free-standing sheets, ${ }^{19,20}$ and free-standing sheets subjected to applied mechanical loading. In the latter category, research has included experiments, ${ }^{21} \mathrm{MD}$ simulations, ${ }^{4,11,12,22}$ and continuum-based approaches ${ }^{13,21,23-27}$ such as finite element simulations and grillage models based on continuum beams. ${ }^{28}$ However, despite this tremendous interest in the topic, only limited research has focused on graphene wrinkling in the context of electronics, ${ }^{16}$ and to the best of our knowledge, none has studied the change in surface area or the effect of defects, both of which are focuses of this work.

At small scales, full atomistic MD simulations using sophisticated force fields are the most reliable way to predict the mechanical response and reveal the physical mechanism of graphene under various loading conditions such as bending, self-folding, crumpling, and stretching up to rupture.,12,22,29 Their application, however, is constrained by sample size limitations, as the method requires large computational resources for force calculation and time integration. ${ }^{27,30,31}$ These limitations motivate the development of multiscale modeling methods that incorporate continuum elasticity theory, which typically use a two-dimensional membrane or shell approximation of a thin sheet. ${ }^{31-34}$ Here, we combine MD

Received: August 12, 2014

Revised: October 4, 2014 
simulations based on rigorous force field with a continuum model based on Koiter's plate theory (KPT). The latter model is found to be rigorous when the bending and stretching energies of a sheet are of comparable magnitude (the regime of interest for deformations involving wrinkling ${ }^{35-37}$ ), and it has been recently successfully applied to study wrinkling in polymeric sheets. $^{33}$

We start by using MD simulations and adopt the Adaptive Intermolecular Reactive Empirical Bond Order (AIREBO) force field to accurately model the interactions among carbon atoms in graphene ${ }^{38,39}$ (the potential equations and boundary conditions are discussed in detail in the Supporting Information). This force field has been widely used and proven to be reliable to reproduce the mechanical behavior observed experimentally in graphene and carbon nanotubes. ${ }^{5,11,12,40} \mathrm{We}$ first investigate the wrinkle pattern in a graphene annulus under torsion, as shown schematically in Figure 1a. For small values of
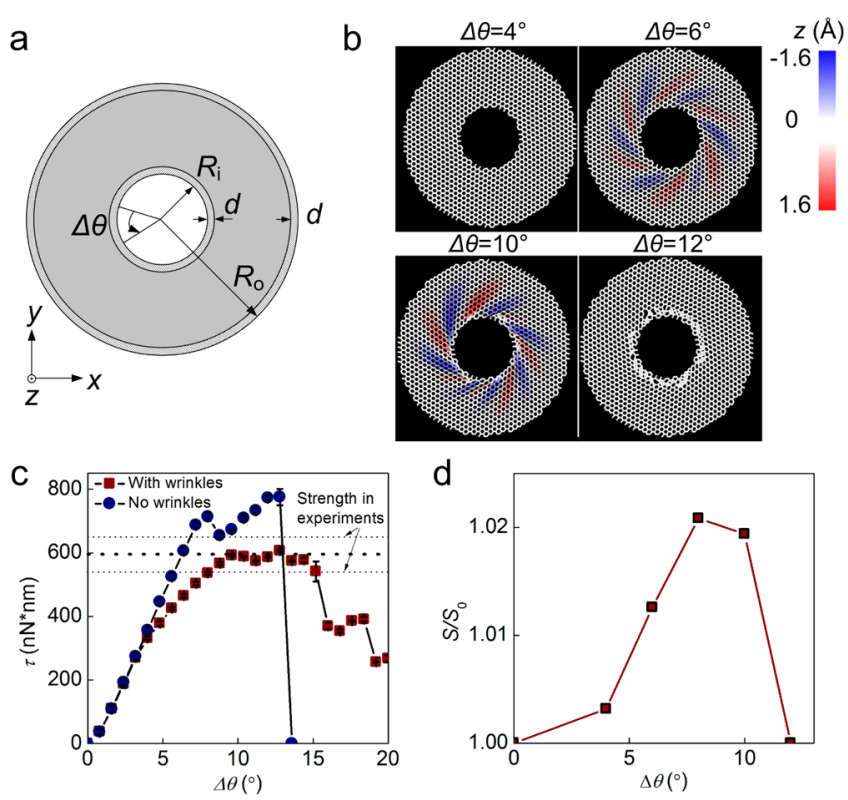

Figure 1. Molecular dynamics (MD) simulations of a graphene annulus with twisted inner boundary. (a) Schematic of boundary conditions used for the graphene annulus. The outer layer of atoms (of width $d$ ) in the annulus is fixed and the inner layer is displaced by a torsion angle of $\Delta \theta$. (b) Simulation snapshots of the equilibrated wrinkled patterns of the graphene annulus for $\Delta \theta=4^{\circ}, 6^{\circ}, 10^{\circ}$, and $12^{\circ}\left(R_{\mathrm{o}} / R_{\mathrm{i}}=3\right.$ and $\left.R_{\mathrm{i}}=1.5 \mathrm{~nm}\right)$. Each atom is colored according to the displacement in the $z$-direction. (c) Total torque on the innermost layer of atoms of the annulus as a function of $\Delta \theta$. For the case with wrinkles, all the atoms between the two boundaries are free to move in all the directions, while for the case of no wrinkles, all the atoms between the two boundaries can only move in the $x-y$ plane. (d) The total surface area (normalized by the projected area $S_{0}$ ) as a function of $\Delta \theta$. Note that even for $\Delta \theta=8-10^{\circ}$, where graphene starts to fail, the surface area change is less than $2 \%$.

torsion angle $\Delta \theta$, no wrinkling is observed. However, when a critical value of $\Delta \theta\left(\Delta \theta \sim 6^{\circ}\right)$ is reached, several wrinkles appear together, as shown in Figure $1 \mathrm{~b}$. When the applied deformation is further increased, the material starts to fail because the covalent bonds on the inner boundary break (see snapshot for $\Delta \theta=10^{\circ}$ in Figure $1 \mathrm{~b}$ ) and, eventually, the wrinkle pattern vanishes (see snapshot for $\Delta \theta=12^{\circ}$ in Figure $1 \mathrm{~b}$ ). We also note that the number of wrinkles, $N$, only slightly increases with the increasing torsion angle $\left(N=6\right.$ for $\Delta \theta=6^{\circ}$ and $N=7$ for $\left.\Delta \theta=10^{\circ}\right)$. At small torsion angles, the wrinkle pattern is regular and resembles a sinusoidal wave along the $\theta$ direction; however, for large torsion angles, irregularities appear because of local bond failure (see Figure S1 in the Supporting Information). We also record the torque measured on the boundary as a function of the applied torsion angle $\Delta \theta$ and compare the results with those obtained for the case where the wrinkles are completely suppressed (in this case the displacements of all the atoms in the $z$-direction are set to be zero). As clearly shown in Figure 1c, the two curves are identical until the critical point is reached. At $\Delta \theta \sim 6^{\circ}$, the two curves start to differ as the case with wrinkles exhibits smaller torque than the case without wrinkles. We also note that both $\tau-\Delta \theta$ curves are characterized by a peak, which reflects the point at which failure first occurs and thus indicates the strength of graphene. For the case with wrinkling, this peak $(\tau=592 \mathrm{nN} \cdot \mathrm{nm})$ occurs at $\Delta \theta=$ $9^{\circ}$. In contrast, a larger torque $(\tau=713 \mathrm{nN} \cdot \mathrm{nm})$ is reached at an earlier stage $\left(\Delta \theta=8^{\circ}\right)$ for the case without wrinkling. Considering the geometry of the annulus, our results predict material strengths of $41.9 \pm 0.3$ and $50.4 \pm 0.9 \mathrm{~N} / \mathrm{m}$ for the cases with and without wrinkles, respectively, which nicely agree with the reported experimental measurements of $42 \pm 4$ $\mathrm{N} / \mathrm{m}^{7}$ (see horizontal dashed lines in Figure 1c). The excellent agreement between our simulation results and the experimental measurements confirms that our MD simulations are appropriate and reliable. Moreover, the better agreement found for the case with wrinkles suggests that wrinkles may be an intrinsic feature of graphene when subjected to loading.

Next, we focus on the effect of wrinkles on the surface area. Although the deformation applied to the annulus is large enough to rupture it, its surface area only increases up to $2 \%$ (see Figure 1d; the calculation of the surface area is discussed in detail in the Supporting Information). This result is very important and suggests that the area-specific capacitance of graphene is not affected by the wrinkle geometry. This observation is also supported by recent experiments that have shown that the capacitance of supercapacitors made of wrinkled graphene sheets is not affected by the applied deformation over hundreds of loading cycles. ${ }^{9}$

We then examine how the surface area of graphene is affected by the system size and use MD simulations to investigate the deformation of graphene annuluses with internal radius $R_{\mathrm{i}}$ ranging from 0.75 to $4.5 \mathrm{~nm}$ (all models are characterized by $R_{\mathrm{o}} / R_{\mathrm{i}}=3, R_{\mathrm{o}}$ denoting the outer radius). Figure $2 \mathrm{a}$ shows the wrinkle patterns calculated at $\Delta \theta=8^{\circ}$. Generally, we find that the wrinkle number $(N)$ is proportional to $R_{\mathrm{i}}$ (Figure $2 \mathrm{~b}$ ), but is almost independent of $R_{\mathrm{o}} / R_{\mathrm{i}}$ (see Table $S 1$ in the Supporting Information), and that larger $R_{\mathrm{i}}$ yield larger out-of-plane fluctuation (see Figure 2c). Moreover, our numerical simulations reveal that the surface area change is not significantly affected by the size of the domain and is always less than $2 \%$ (Figure $2 \mathrm{~d}$ ). Interestingly, the effect of size on the out-of-plane fluctuations can be nicely captured using a simple geometric model that assumes a sinusoidal profile along the $\theta$ direction and describes the height of the wrinkles with a lognormal function (see Figure S2; the geometric model is discussed in detail in the Supporting Information). However, the simple geometric model cannot well predict the change of surface area induced by the wrinkles (see continuous black line in Figure 2d). One of the reasons for this difference is that a graphene wrinkle at large deformation is not exactly sinusoidal in shape, such that the geometric model has limited application for surface area predictions. 

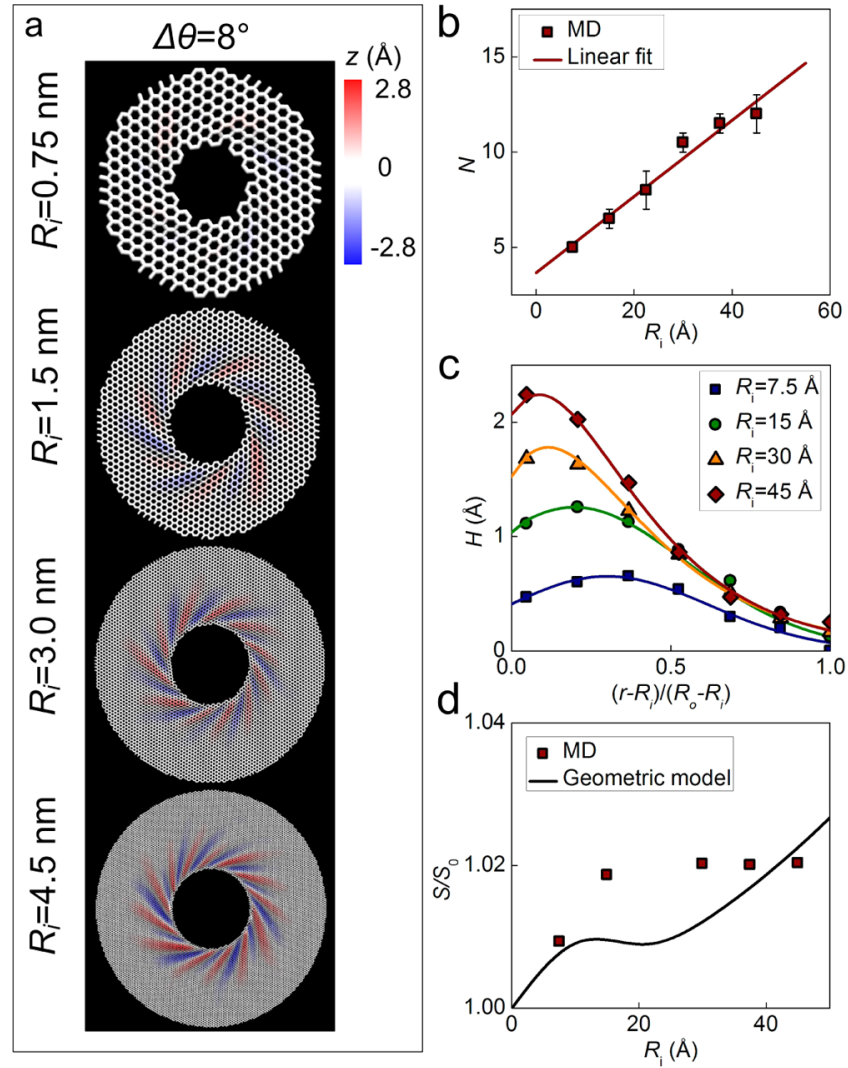

Figure 2. Effect of size on the surface area of a wrinkled graphene annulus. (a) Snapshots taken for several graphene annuluses under a torsion angle of $\Delta \theta=8^{\circ}$ in MD simulations $\left(R_{\mathrm{o}} / R_{\mathrm{i}}=3\right.$ and $R_{\mathrm{i}}=0.75$, $1.5,3$, and $4.5 \mathrm{~nm}$ ). (b) The number of wrinkles as a function of $R_{\mathrm{i}}$ for annuluses with $R_{\mathrm{o}} / R_{\mathrm{i}}=3$. The effect of $\Delta \theta$ is shown via error bars representing standard deviation. The solid curve is given by a linear fit. (c) The amplitude of wrinkles as a function of distance from the inner boundary. Curves are given for the different $R_{\mathrm{i}}$ shown in (a). The solid curves are generated using the geometric model given in Supporting Information eq S3 with associated parameters provided in Supporting Information Table S1. The data points are measured from MD simulations. (d) The total surface area as a function of $R_{\mathrm{i}}$ under torsion of $\Delta \theta=8^{\circ}\left(R_{\mathrm{o}} / R_{\mathrm{i}}=3\right)$. The geometric model overpredicts the area change for large graphene annuluses.

To overcome the limitations of the geometric model and to facilitate investigations of wrinkling in larger domains, we assumed that the mechanism of bending resistance in a graphene monolayer is reasonably well-described by the mechanism that characterizes bending resistance in a thin continuum shell and developed a graphene model based on KPT (the model is discussed in detail in the Supporting Information). To estimate the bending stiffness to be entered into the model, we performed a full atomistic simulation of a rectangular graphene sheet with one edge clamped and the other subjected to a transverse displacement $\delta$ (see Figure 3a). The force $(F)$-deflection $(\delta)$ relation as measured in MD simulations is shown in Figure $3 \mathrm{~b}$. Since according to beam theory $^{41}$

$$
F=12 D / L^{2}(\delta / L)
$$

(where $L=3.1 \mathrm{~nm}$ is the effective length of the plate), the numerical results reported in Figure $3 \mathrm{~b}$ can be used to calculate the bending stiffness $D$. It is important to note that, as indicated by the slope of the curve, $D$ changes as a function of the applied a
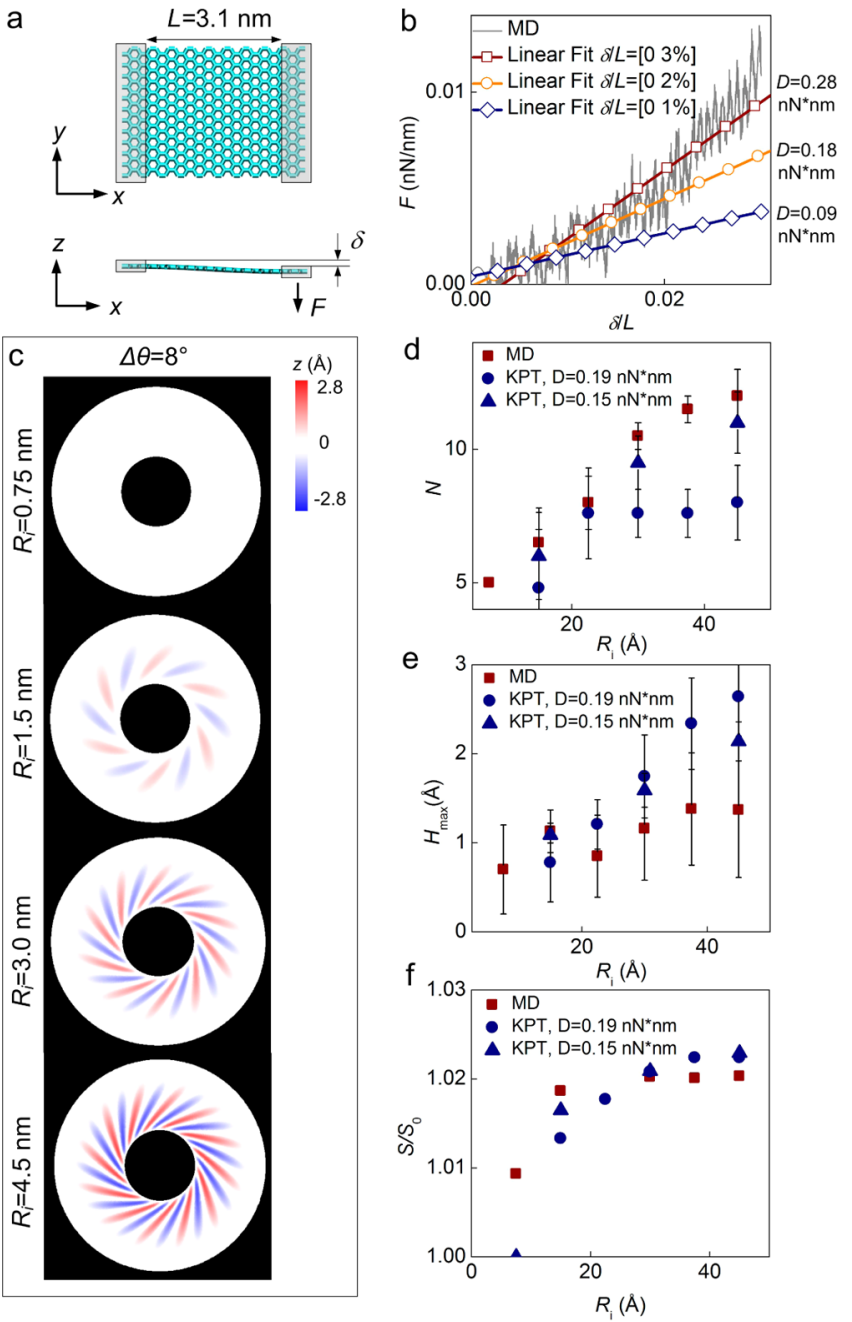

Figure 3. Comparison between the results given by $\mathrm{MD}$ simulations and a continuum mechanics model based on KPT. (a) Schematic of the model deformation used to measure the effective KPT bending stiffness via $\mathrm{MD}$ simulation. (b) The force-displacement curve obtained for the model shown in panel a from MD simulation. Three measurements of the slope of the loading curve are obtained based on a linear fit from $\delta / L=0$ to $1 \%, 2 \%$, and 3\% displacement, respectively. (c) Snapshots for results given by the KPT model for annuluses with $R_{\mathrm{o}} / R_{\mathrm{i}}=3$ and $R_{\mathrm{i}}=0.75,1.5,3$, and $4.5 \mathrm{~nm}$ under a torsion angle of $\Delta \theta$ $=8^{\circ}$, which show good agreement with the corresponding MD results in Figure 2a. (d) Comparison between the MD and KPT results for the number of wrinkles as a function of $R_{\mathrm{i}}$ KPT results are provided for two different values of effective bending stiffness. The effect of $\Delta \theta$ is included in the error bar representing standard deviation. (e) Comparison between the MD and KPT results for the wrinkle amplitude as a function of $R_{\mathrm{i}}$. Error bars again represent standard deviation and show the effect of changing $\Delta \theta$. (f) The total surface area as a function of $R_{\mathrm{i}}$ for the fixed $R_{\mathrm{o}} / R_{\mathrm{i}}=3$ under torsion of $\Delta \theta=$ $8^{\circ}$. The KPT model using a smaller bending stiffness, $D=0.15 \mathrm{nN} \cdot \mathrm{nm}$, gives better agreement with the MD results in panels $\mathrm{d}-\mathrm{f}$.

deformation. In fact, larger deflection yields larger $D$, which is found to vary between $0.09 \mathrm{nN} \cdot \mathrm{nm}$ (for $\delta / L=0.01$ ) and 0.28 $\mathrm{nN} \cdot \mathrm{nm}$ (for $\delta / L=0.03$ ). This result agrees with theoretical predictions of graphene's bending stiffness in folding $\left(D_{\text {fold }}=\right.$ $0.19 \mathrm{nN} \cdot \mathrm{nm})^{11}$ and also explains the large variation of bending stiffness that are used by different groups to build continuum models of graphene. ${ }^{1,42-44}$ Having determined a range of values for the bending stiffness of graphene, we now assume $D$ 

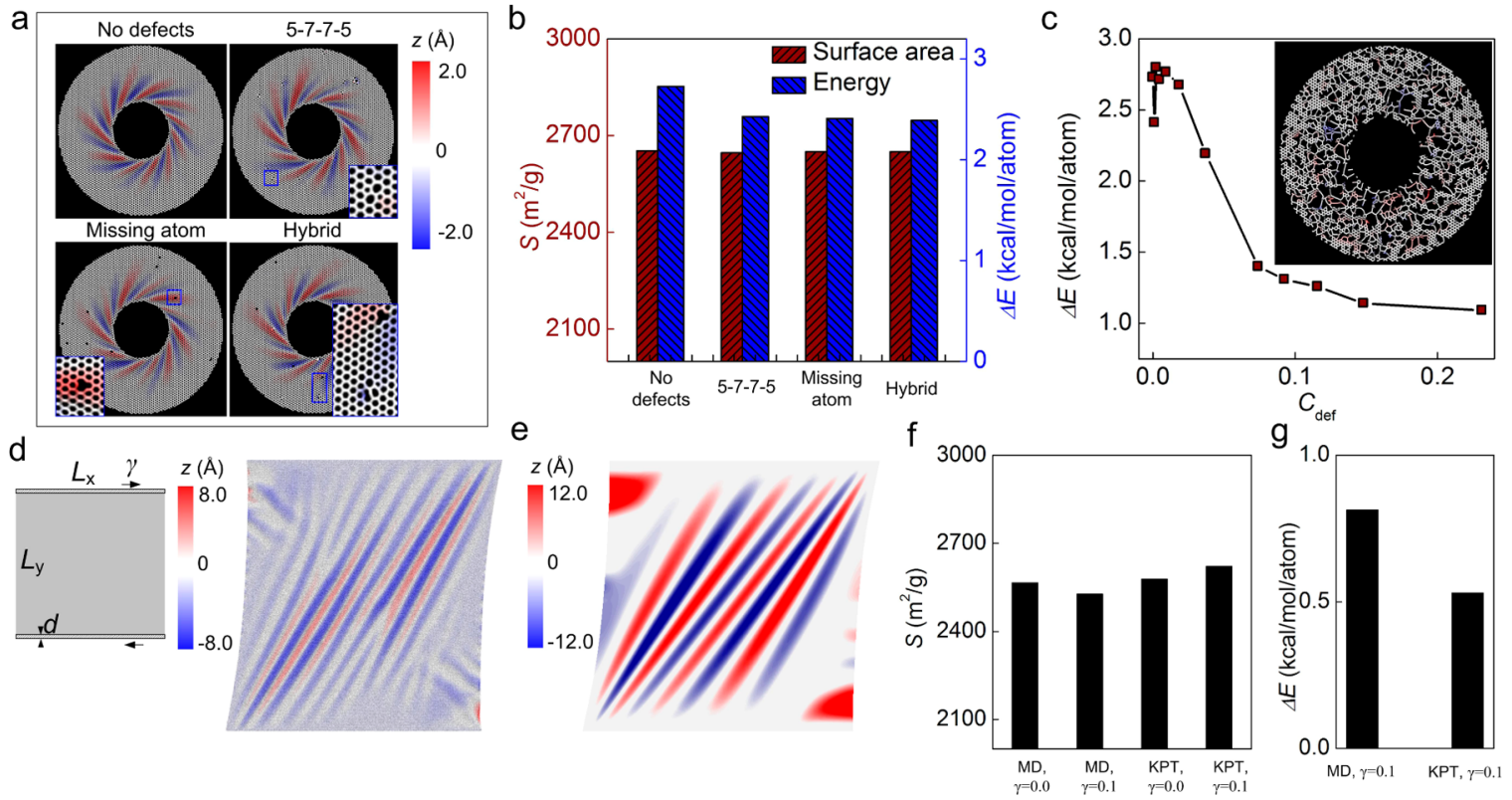

Figure 4. Comparison of graphene surface area for varied defects and geometries. (a) MD simulation snapshots of a graphene annulus $\left(R_{\mathrm{o}} / R_{\mathrm{i}}=3\right.$ and $R_{\mathrm{i}}=3 \mathrm{~nm}$ ) with $\Delta \theta=8^{\circ}$ and different forms of defects (pristine graphene, graphene with 10 5-7-7-5 defects, graphene with 10 missing atoms, and graphene with 5 missing atoms and 5 5-7-7-5 defects). In all models before randomly creating defects we have 8642 atoms, so that the defect concentration is $C_{\mathrm{def}}=0.001$. (b) Effect of defects on the average surface area (left axis, surface area averaged by the graphene mass) and energy caused by deformation ( $\Delta E$, right axis) for the multiple defected systems in a. Note that all the results are close to $2590 \mathrm{~m}^{2} / \mathrm{g}$ (the value for pristine graphene at equilibrium) without deformation or wrinkles. (c) $\Delta E$ as a function of the defect concentration $\left(C_{\text {def }}\right)$ for the graphene annulus. Inserted snapshot is for a graphene annulus with $C_{\mathrm{def}}=0.23$ for $\Delta \theta=8^{\circ}$, showing that the wrinkle pattern in completely suppressed (the color of the atoms corresponds to the same color bar as given in panel a). (d) The schematics of the boundary conditions used for the graphene rectangle and the MD simulation snapshot of the wrinkled pattern of a large rectangular graphene sheet $\left(L_{x}=L_{y}=77 \mathrm{~nm}\right)$ under a shearing strain of $\gamma=0.1$. (e) Using the same geometry and boundary conditions as $\mathrm{c}$, we repeat the simulation using the KPT method and take the snapshot shown. (f) Comparison of the graphene surface area for the different geometries and simulation methods. Again, it is noted that all the results are close to $2590 \mathrm{~m}^{2} / \mathrm{g}$. $(\mathrm{g})$ Comparison of $\Delta E$ for the system as shown in $\mathrm{c}$ and $\mathrm{d}$ that are obtained from the MD and KPT simulations, respectively.

to be a constant and use different values within the measured range in KPT model to investigate the formation of wrinkles in an annulus subjected to torsion. In Figure $3 c$, we report the wrinkle patterns observed at $\Delta \theta=8^{\circ}$ assuming the effective bending stiffness $D_{\text {eff }}=0.15 \mathrm{nN} \cdot \mathrm{nm}$ for annuluses with the same dimensions as those shown in Figure 2a. Clearly, the continuum model gives much better agreement with $\mathrm{MD}$ simulations (Figure 2a) than the simple geometric model (see Figure S2 in Supporting Information), and correctly captures not only the number of wrinkles, but also their morphology. A quantitative comparison between the numerical results obtained using MD and the continuum model is shown in Figure $3 \mathrm{~d}-\mathrm{f}$, where we report the evolution of $N, H_{\max }$ and the surface area as a function of the annulus size. Good agreement between $\mathrm{MD}$ and KPT simulations is found using $D_{\text {eff }}$ in the continuum model. However, as shown in Figure 3d,e, using the larger bending stiffness $D_{\text {fold }}$ (i.e., the theoretical graphene folding result) instead yields fewer wrinkles and larger amplitudes, and thus, a worse agreement with $\mathrm{MD}$. The better agreement for KPT against MD simulations by using $D_{\text {eff }}(0.15$ $\mathrm{nN} \cdot \mathrm{nm})$ rather than $D_{\text {fold }}(0.19 \mathrm{nN} \cdot \mathrm{nm})$ suggests that the deformation in graphene wrinkling in the current study is smaller than that in graphene folding in literature. ${ }^{11}$ In addition, Figure $3 \mathrm{f}$ shows that the KPT simulations can accurately predict the evolution of the surface area, in contrast to the geometric model (Figure $2 \mathrm{~d}$ ). We find that the KPT simulations provide more computing efficiency than $\mathrm{MD}$, particularly at larger length scales.
Since the properties of graphene are known to be highly affected by intrinsic defects, ${ }^{45}$ we also used MD simulations to investigate the effect of various kinds of defects including 5-7-75 (i.e., Stone-Wales defects, a defect with one $\mathrm{C}-\mathrm{C}$ bond rotated in plane for $90^{\circ}$ ), missing atom and a hybrid of both types on the wrinkled patterns. As shown in Figure 4a, for low concentration of defects $\left(C_{\mathrm{def}}=\right.$ (number of defects) $/$ (total number of atoms in pristine model $)=0.001)$, the defects do not alter the overall wrinkle pattern (both $N$ and $H_{\max }$ are almost constant for the 4 models shown in in Figure 4a). We also computed and compared the surface area of systems with and without defects. The results, summarized in Figure $4 b$, show that for all configurations with $C_{\mathrm{def}}=0.001$ the surface area is close to the theoretical value of planar graphene $\left(S_{0}=\right.$ $\left.2600 \mathrm{~m}^{2} / \mathrm{g}\right),{ }^{8}$ and is not affected by the defects and that defects can only cause less than $0.2 \%$ difference in surface area. We also compared the total potential energy of the undeformed $\left(E_{0}\right.$, for $\Delta \theta=0^{\circ}$, no wrinkles $)$ and wrinkled $\left(E_{1}\right.$, for $\left.\Delta \theta=8^{\circ}\right)$ configurations and computed the energy change $\left(\Delta E=E_{1}-\right.$ $\left.E_{0}\right)$ for each carbon atom, as shown in Figure $4 \mathrm{~b}$. Again, we note that the values obtained for the models with defects $(2.41$ $\pm 0.02 \mathrm{kcal} /(\mathrm{mol} /$ atom $)$ for the 3 models with defects $)$ are close to those reported for pristine graphene $(2.73 \mathrm{kcal} /(\mathrm{mol} /$ atom)), suggesting that low concentrations of defects do not alter the overall deformation field in graphene. Next, we investigated how the wrinkle pattern and surface area is affected by the defect concentration in the graphene annulus. Starting from $C_{\text {def }}=0.001$, we progressively increase the concentration of defects up to $C_{\mathrm{def}}=0.23$ and measure $\Delta E$ for $\Delta \theta=8^{\circ}$. The 
results shown in Figure $4 c$ clearly indicate the existence of a critical threshold: for $C_{\text {def }}<0.04$, both the wrinkle pattern and the surface area of the sheet are only marginally altered; on the other hand, if $C_{\text {def }}>0.04, \Delta E$ gets significantly reduced and the deformation of the sheet gets significantly altered. In fact, in graphene with high defect concentration, the out-of-plane wrinkle pattern is completely suppressed (see inset in Figure 4c) and the deformation energy is released in-plane by packing and merging of the defects.

Finally, we investigated the effect of geometry and loading conditions on the change of surface area induced by wrinkles. To this end, we considered a rectangle domain loaded in shear (as shown in Figure $4 \mathrm{~d}$, the bottom layer of atoms is fixed and top edge is displaced in $x$-direction with shear strain $\gamma=0.1$ ). As reported in Figure 4d,e, we find good qualitative agreement between the wrinkling patterns obtained in MD and KPT simulations using $D=D_{\text {eff. }}$ We note that the KPT results show fewer wrinkles at larger amplitudes than the MD results, indicating that the effective bending stiffness of graphene for this loading case is slightly lower. Interestingly, also for this configuration and loading conditions, we find that the surface area of the graphene does not vary much from its relaxed state (within $\pm 1.7 \%$ for both MD and KPT simulations), as shown in Figure 4f. Moreover, in Figure $4 \mathrm{~g}$, we report the change in energy measured both in $\mathrm{MD}(\Delta E=0.81 \mathrm{kcal} /(\mathrm{mol} /$ atom $))$ and $\mathrm{KPT}(0.53 \mathrm{kcal} /(\mathrm{mol} /$ atom $))$ simulations. Note that the overall smaller deformation energy obtained from the continuum model is due to the fact that these simulations do not account for van der Waals interactions that become important as adjacent wrinkle surfaces come into close proximity with each other in large deformations (stacking conformation). ${ }^{46}$

We used MD and continuum mechanics-based simulations to study wrinkling in free-standing graphene. In particular, we investigated the effect of deformations involving wrinkling on the surface area. We have shown that the large specific surface area of graphene $\left(2590 \mathrm{~m}^{2} / \mathrm{g}\right)$ does not vary beyond $2 \%$ for a variety of loading and geometries, a result that agrees with experimental observations. ${ }^{9}$ We have also demonstrated that this result is not affected by defects (if $C_{\mathrm{def}}<0.04$ ), geometry, and loading conditions. In addition, we observe that the number and amplitude of the wrinkles are overall proportional to the sample size, but their exact numerical value depends on the deformation amount. To our knowledge, this is the first time that the effect of loading conditions, amount of loading and defects on the surface area of graphene is rigorously demonstrated. This is an issue of great importance and our results provide important tools for the design of the next generation of graphene nanoelectronics.

We have also demonstrated that continuum simulations using KPT are capable of providing excellent agreement with $\mathrm{MD}$ results provided a suitable effective bending stiffness is used. Our simulations clearly indicate that this stiffness is dependent on the amount and type of applied loading. In fact, we found that for an annulus under torsion an effective bending stiffness of $0.15 \mathrm{nN} \cdot \mathrm{nm}$ works well. However, this value should likely be lower for the shearing of a rectangular graphene sheet. To our knowledge, the application of KPT to graphene deformation is novel. Since such model is suitable for simulating graphene sheets across a wide range of length scales, it is vitally important for problems too large to be efficiently simulated by $\mathrm{MD}$, which may be the case in many potential nanoelectronic applications. Improving the model, such as by adding nonbonded interaction derived from full atomistic $\mathrm{MD}$, is a rich avenue for future investigation.

Consistent large surface area, combined with high strength, makes graphene an ideal material for constructing supercapacitors with long life, high capacitance, and stability for application in energy storage as well as flexible nanoelectronic circuits. With the models developed in this work, we provide important insight into the deformation of graphene as well as tools for designing the next generation of graphene nanoelectronics.

\section{ASSOCIATED CONTENT}

\section{Supporting Information}

Molecular dynamics simulations method, boundary conditions and method to calculate surface area from full atomistic model, geometric model of wrinkle patterns, continuum mechanics model using Koiter's nonlinear plate theory, Table S1. This material is available free of charge via the Internet at http:// pubs.acs.org.

\section{AUTHOR INFORMATION}

\section{Corresponding Authors}

*E-mail: bertoldi@seas.harvard.edu.

*E-mail: mbuehler@mit.edu.

\section{Author Contributions}

${ }^{\#}$ Z.Q. and M.T. contributed equally to this work.

\section{Notes}

The authors declare no competing financial interest.

\section{ACKNOWLEDGMENTS}

Z.Q., M.H. and M.J.B. acknowledge support from ONRPECASE, AFOSR, and ARO as well as the MIT UROP Office. K.B. and M.J.T. acknowledge startup funds from the School of Engineering and Applied Sciences, Harvard University and the support of the Kavli Institute at Harvard University.

\section{REFERENCES}

(1) Geim, A. K.; Kim, P. Sci. Am. 2008, 298, 90-97.

(2) Geim, A. K.; Novoselov, K. S. Nat. Mater. 2007, 6, 183-191.

(3) Meyer, J. C.; Geim, A. K.; Katsnelson, M. I.; Novoselov, K. S.; Booth, T. J.; Roth, S. Nature 2007, 446, 60-63.

(4) Sen, D.; Novoselov, K. S.; Reis, P. M.; Buehler, M. J. Small 2010 , 6, 1108-1116.

(5) Qin, Z.; Feng, X. Q.; Zou, J.; Yin, Y. J.; Yu, S. W. Appl. Phys. Lett. 2007, 91, 043108.

(6) Qin, Z.; Buehler, M. J. Journal of Applied Mechanics 2013, dx.doi. org/10.1115/1.4023641.

(7) Lee, G. H.; Cooper, R. C.; An, S. J.; Lee, S.; van der Zande, A.; Petrone, N.; Hammerherg, A. G.; Lee, C.; Crawford, B.; Oliver, W.; Kysar, J. W.; Hone, J. Science 2013, 340, 1073-1076.

(8) Kuchta, B.; Firlej, L.; Mohammadhosseini, A.; Boulet, P.; Beckner, M.; Romanos, J.; Pfeifer, P. J. Am. Chem. Soc. 2012, 134, 15130-15137.

(9) Chen, T.; Xue, Y. H.; Roy, A. K.; Dai, L. M. ACS Nano 2014, 8, 1039-1046.

(10) Wood, B. C.; Ogitsu, T.; Otani, M.; Biener, J. J. Phys. Chem. C 2014, 118, 4-15.

(11) Cranford, S.; Sen, D.; Buehler, M. J. Appl. Phys. Lett. 2009, 95.

(12) Xu, Z. P.; Buehler, M. J. ACS Nano 2010, 4, 3869-3876.

(13) Wang, J. W.; Cao, Y. P.; Feng, X. Q. Appl. Phys. Lett. 2014, 104.

(14) Ko, H. C.; Stoykovich, M. P.; Song, J. Z.; Malyarchuk, V.; Choi, W. M.; Yu, C. J.; Geddes, J. B.; Xiao, J. L.; Wang, S. D.; Huang, Y. G.; Rogers, J. A. Nature 2008, 454, 748-753.

(15) El-Kady, M. F.; Kaner, R. B. Nat. Commun. 2013, 4. 
(16) Zhu, W. J.; Low, T.; Perebeinos, V.; Bol, A. A.; Zhu, Y.; Yan, H.

G.; Tersoff, J.; Avouris, P. Nano Lett. 2012, 12, 3431-3436.

(17) Pan, Z. H.; Liu, N.; Fu, L.; Liu, Z. F. J. Am. Chem. Soc. 2011, 133, 17578-17581.

(18) Liu, N.; Pan, Z. H.; Fu, L.; Zhang, C. H.; Dai, B. Y.; Liu, Z. F. Nano Res. 2011, 4, 996-1004.

(19) Thompson-Flagg, R. C.; Moura, M. J. B.; Marder, M. EPL 2009, 85.

(20) Shenoy, V. B.; Reddy, C. D.; Ramasubramaniam, A.; Zhang, Y. W. Phys. Rev. Lett. 2008, 101.

(21) Bao, W. Z.; Miao, F.; Chen, Z.; Zhang, H.; Jang, W. Y.; Dames, C.; Lau, C. N. Nat. Nanotechnol. 2009, 4, 562-566.

(22) Zhang, P.; Ma, L. L.; Fan, F. F.; Zeng, Z.; Peng, C.; Loya, P. E.; Liu, Z.; Gong, Y. J.; Zhang, J. N.; Zhang, X. X.; Ajayan, P. M.; Zhu, T.; Lou, J. Nat. Commun. 2014, 5.

(23) Gil, A. J.; Adhikari, S.; Scarpa, F.; Bonet, J. J. Phys.: Condens. Matter 2010, 22.

(24) Vandeparre, H.; Pineirua, M.; Brau, F.; Roman, B.; Bico, J.; Gay, C.; Bao, W. Z.; Lau, C. N.; Reis, P. M.; Damman, P. Phys. Rev. Lett. 2011, 106.

(25) Wang, C. G.; Lan, L.; Tan, H. F. Phys. Chem. Chem. Phys. 2013, $15,2764-2773$.

(26) Wang, C. Y.; Mylvaganam, K.; Zhang, L. C. Phys. Rev. B 2009, 80.

(27) Duan, W. H.; Gong, K.; Wang, Q. Carbon 2011, 49, 31073112.

(28) Zhang, Z.; Duan, W. H.; Wang, C. M. J. Appl. Phys. 2013, 113.

(29) Cranford, S. W.; Buehler, M. J. Phys. Rev. B 2011, 84.

(30) Zhang, Z.; Duan, W. H.; Wang, C. M. Nanoscale 2012, 4, 50775081.

(31) Zheng, Q. B.; Geng, Y.; Wang, S. J.; Li, Z. G.; Kim, J. K. Carbon 2010, 48, 4315-4322.

(32) Nayyar, V.; Ravi-Chandar, K.; Huang, R. Int. J. Solids Struct. 2011, 48, 3471-3483.

(33) Taylor, M.; Bertoldi, K.; Steigmann, D. J. J. Mech. Phys. Solids 2014, 62, 163-180.

(34) Wong, Y. W.; Pellegrino, S. J. Mech. Mater. Struct. 2006, 1, 6395.

(35) Steigmann, D. J. In Poly-, Quasi- and Rank-One Convexity in Applied Mechanics; Schröder, J.; Neff, P.; CISM Courses and Lectures No. 516; Springer: New York, 2010; pp 265-299.

(36) Steigmann, D. J. Math. Mech. Solids 2013, 18, 103-112.

(37) Steigmann, D. J. Journal of Elasticity 2013, 111, 91-107.

(38) Stuart, S. J.; Tutein, A. B.; Harrison, J. A. J. Chem. Phys. 2000, 112, 6472-6486.

(39) Brenner, D. W.; Shenderova, O. A.; Harrison, J. A.; Stuart, S. J.; Ni, B.; Sinnott, S. B. J. Phys.: Condens. Matter 2002, 14, 783-802.

(40) Wei, Y. J.; Wu, J. T.; Yin, H. Q.; Shi, X. H.; Yang, R. G.; Dresselhaus, M. Nat. Mater. 2012, 11, 759-763.

(41) Gere, J. M.; Timoshenko, S. Mechanics of Materials, 4th ed.; PWS Pub Co.: Boston, MA, 1997; p xvi.

(42) Lu, Q.; Huang, R. Int. J. Appl. Mech. 2009, 1, 443-467.

(43) Arroyo, M.; Belytschko, T. Phys. Rev. B 2004, 69.

(44) Kudin, K. N.; Scuseria, G. E.; Yakobson, B. I. Phys. Rev. B 2001, 64.

(45) Banhart, F.; Kotakoski, J.; Krasheninnikov, A. V. ACS Nano 2011, 5, 26-41.

(46) Lui, C. H.; Li, Z. Q.; Chen, Z. Y.; Klimov, P. V.; Brus, L. E.; Heinz, T. F. Nano Lett. 2011, 11, 164-169. 


\section{SUPPORTING INFORMATION:}

\section{Effect of wrinkles on the surface area of graphene: toward the design of nanoelectronics}

Zhao Qin $^{1,2 \#}$, Michael Taylor ${ }^{3 \#}$, Mary Hwang ${ }^{1,2}$, Katia Bertoldi ${ }^{3,4 *}$ and Markus J. Buehler ${ }^{1,2 *}$

\section{$\underline{\text { Molecular dynamics simulations }}$}

MD simulations are performed via LAMMPS MD package ${ }^{1}$ using the Adaptive Intermolecular Reactive Empirical Bond Order (AIREBO) force field to model the internal interactions among carbon atoms in graphene ${ }^{2,3}$. This has been widely used and proven to be a reliable force field to reproduce the mechanical behavior observed experimentally in graphene and carbon nanotube ${ }^{4-7}$. According to this model the total potential energy of the system is given by:

$E=\frac{1}{2} \sum_{i} \sum_{j \neq i}\left[E_{i j}^{R E B O}+\sum_{k \neq i} \sum_{l \neq i, j, k} E_{k i j l}^{T O R S I O N}\right]$

which accounts for all interactions among carbon atoms in graphene. Moreover, $E_{i j}^{R E B O}$ denotes the REBO term of hydrocarbon ${ }^{3}$ and $E_{k i j l}^{T O R S I O N}$ is an explicit 4-body potential that describes various dihedral angle preferences in hydrocarbon configurations. The detailed expressions for $E_{i j}^{R E B O}$ and $E_{k i j l}^{T O R S I O N}$ are given in the original paper ${ }^{2}$.

We apply a $N V T$ ensemble to ensure constant volume and temperature for all simulations and the temperature of each simulation is controlled by a Nose-Hoover thermostat. Moreover, each combination of initial geometry and loading condition on the boundary is investigated by an independent simulation.

Energy minimization of 10000 steps by using the conjugate gradient algorithm is applied to the system prior to dynamics simulation. We compute the root-mean-square deviation and total energy of the system during dynamics simulation for equilibrium calculation for 500 ps (with 1 fs timestep) and ensure their convergence before the end of each simulation. By doing so, we confirm that the simulation is sufficiently long to capture the equilibrated conformation of wrinkled graphene. We record the coordinates of all the atoms at equilibrium and count the number of wrinkles. We have tried different thermostat temperatures and random velocity distributions (initial temperature) to confirm that the result is not sensitive to those factors.

\section{Geometry and boundary conditions}

Two geometries are used here to investigate the effect of wrinkling:

(i) A graphene annulus (with inner and outer radii $R_{i}$ and $R_{o}$, respectively ) subjected to torsion (see Fig. 1a)

(ii) A rectangular graphene sheet (with edges $L_{x}$ and $L_{y}$ ) under shearing (see Fig. 4c)

For all simulations, we start by displacing all atoms in such a way that the desired level of 
displacement is applied to the boundaries and then use MD to equilibrate the structure. For the annulus all atoms are given an initial angular displacement $\Delta \theta \frac{r-R_{i}}{R_{0}-R_{i}}$, where $r$ is the distance of the atom to the center of the annulus and $\Delta \theta$ is the applied torsion angle applied to the inner boundary. Differently, for the rectangular sheet all atoms are given an horizontal displacement $\gamma y$, where $\gamma$ is the amount of applied strain and $y$ is the distance of the atom from the boundary. Note that all the atoms within a distance $d=2 \AA$ from the boundaries where the displacements are applied (i.e. inner and outer perimeter of the annulus and bottom and top edges of the rectangle) are fixed throughout the entire equilibration process.
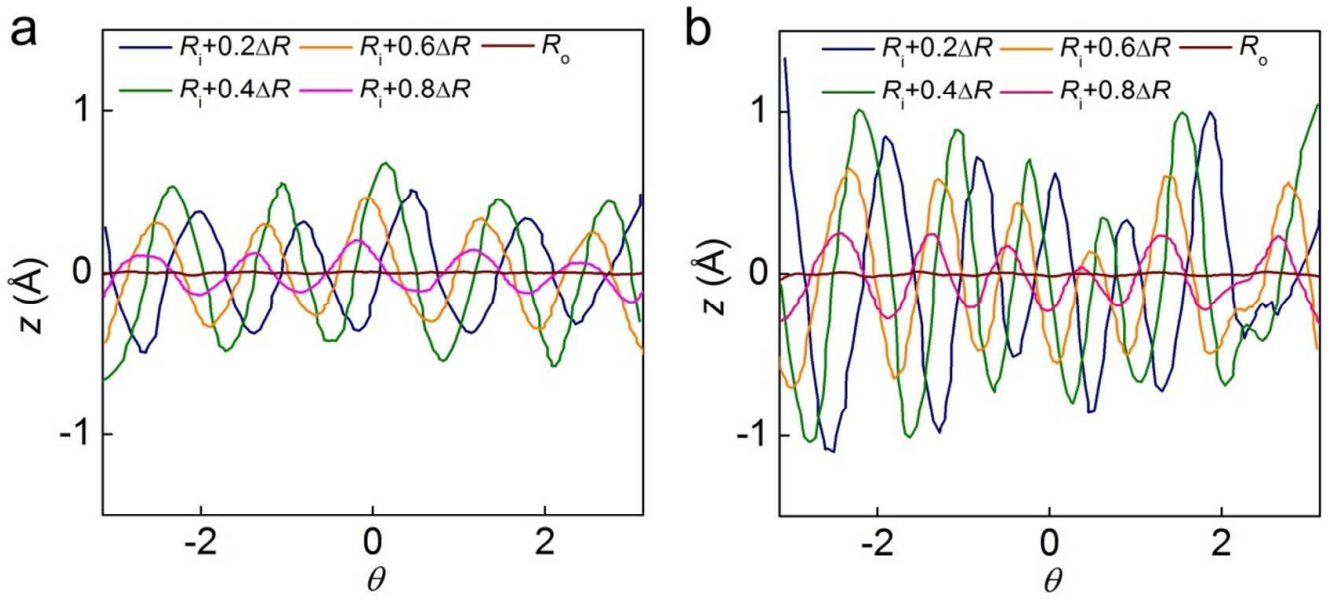

Figure S1. Out-of-plane deformation of a graphene annulus subjected to torsion. Out-of-plane displacements along circumferential paths at different distance from the center (in this case $R_{o} /$ $R_{i}=3$ and $R_{i}=1.5 \mathrm{~nm}$ ) for $\Delta \theta=6^{\circ}$ in $\mathbf{a}$ and $\Delta \theta=10^{\circ}$ in $\mathbf{b}$. The results are obtained using MD.

\section{Surface area calculation for the full atomistic model}

To compute the total surface area of grapheQe at different levels of deformation we use the equilibrated structure. in and the Delaunay triangulation algorithm ${ }^{8}$ to generate a triangular mesh based on the location of the atoms. We carefully select the cut-off distance ( $3 \AA$ as the longest distance within an aromatic ring) to generate the triangle mesh, to make sure that we do not overestimate the atomic area at the boundaries. The total surface area is then calculated as the sum of the area of each triangle, which is given by

$s=\left|\overrightarrow{l_{1}} \times \overrightarrow{l_{2}}\right|$

where $\overrightarrow{l_{1}}$ and $\overrightarrow{l_{2}}$ are the vectors spanning two edges of the triangle.

\section{Ceometric model of wrinkle patternsin an_annulus}

Since our MD results indicate that the out-of-plane displacement $(z)$ along the $\theta$ direction can be well approximated by a sinusoidal function, we assume that $z(\theta, r)$ for an atom with coordinate $(\theta, r)$ is given by

$z(\theta, r)=H\left(r, R_{i}, R_{o}\right) \sin \left[N \theta+\frac{\pi\left(r-R_{i}\right)}{2\left(R_{o}-R_{i}\right)}\right]$

where $H\left(r, R_{i}, R_{o}\right)$ is the height of the wrinkle at a distance $r$ from the annulus center, $R_{i}$ and $R_{o}$ 
define the inner and outer radii of the annulus, and $N$ is the number of wrinkles.

Moreover, the MD results reported in Fig. 2c of the main text suggest that the height of the wrinkles can be captured using a log-normal function,

$H\left(r, R_{i}, R_{o}\right)=H_{\max } \exp \left\{-\left[\ln \left(\frac{r-R_{i}}{R_{o}-R_{i}}+C\right)-A\right]^{2} \frac{1}{2 B^{2}}\right\}$

Where $H_{\max }$ is the maximum out-of-plane displacement of all the carbon atoms, and $A, B$ and $C$ are constants determined by fitting the equation to our MD results, as summarized in Table S1. The wrinkle patterns obtained using this simple geometric model are shown in Fig. S2 for grapheQ annuluses with internal radius $R_{i}$ ranging from 0.75 to $4.5 \mathrm{~nm}$ (all models are characterized by $R_{o} / R_{i}=3, R_{o}$ denoting the outer radius). For all cases the wrinkle patterns are calculated at $\Delta \theta=8^{\circ}$. Finally, the total surface area can be easily calculated as

$S\left(R_{o}, R_{i}, \Delta \theta\right)=\int_{R_{i}}^{R_{0}} \int_{0}^{2 \pi} r \sqrt{\left(\frac{\partial \mathrm{z}}{r \partial \theta}\right)^{2}+\left(\frac{\partial \mathrm{z}}{\partial r}\right)^{2}+1} \cdot d \theta d r$
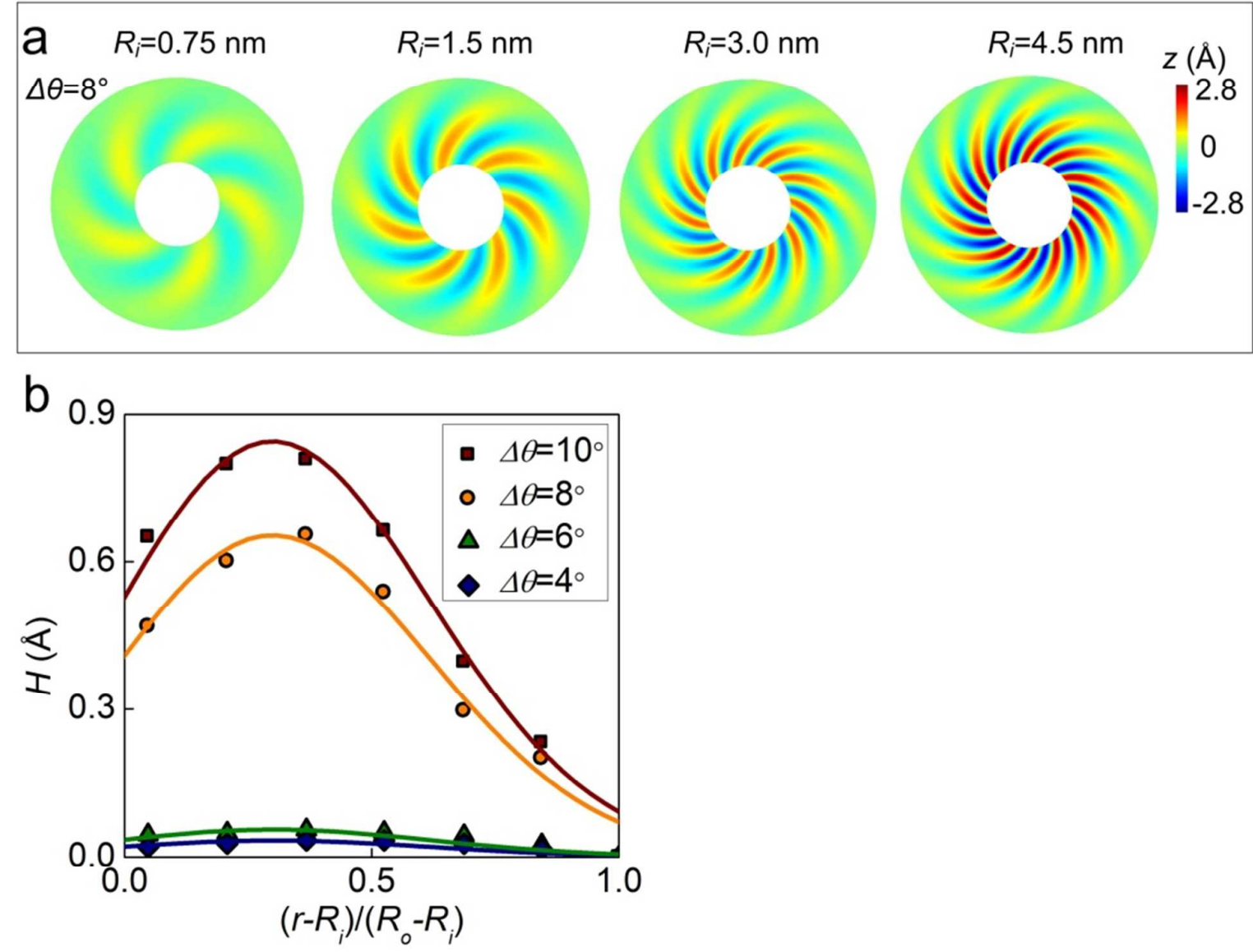

Figure S2. Wrinkle pattern predicted by the geometric model. a. Wrinkle patternVpredicted by the geometric model (Eq. (S2) and Table S1) for graphene annuli with $R_{o} / R_{i}=3$ and $R_{i}=$ $0.75,1.5,3$ and $4.5 \mathrm{~nm}$ subjected to a torsion angle of $\Delta \theta=8^{\circ}$. b. Amplitude of out-of-plane displacement $(H)$ for atoms at different distance $(r)$ from the annulus center $\left(R_{o} / R_{i}=3\right.$ and $R_{i}=0.75 \mathrm{~nm}$ ). Markers and continuous lines correspond to MD results and geometric model predictions, respectively. $\theta$.

\section{Simulations using Koiter's non-linear plate theory via dynamic relaxation}

In this section, we give a very brief summary of the theory and methods underpinning the 
continuum simulations. Detailed information can be found in ${ }^{9}$. We note that in applying a continuum model to graphene an important assumption is made. That is, we assume the mechanism of bending resistance in a graphene mono-layer is reasonably well-described by the mechanism that characterizes bending resistance in a thin continuum shell. The nature of these mechanisms is, in fact, quite different as a continuum shell derives its bending resistance from having a finite thickness through which a portion is in tension and the rest is in compression during a bending deformation ${ }^{10}$. Graphene, being one atomic layer thick derives its bending stiffness directly from stretching of atomic bonds. The concept of thickness, in a continuum sense, of a single atom is not well defined.

\section{Plate model}

The equilibrium equations of the midsurface, $\Omega$, of an initially flat sheet are given by, $\operatorname{div} \mathbf{T}=\mathbf{0}$ or $T_{i \alpha, \alpha}=0$,

where the $1^{\text {st }}$ Piola-Kirchoff stress, $\mathbf{T}$, has components

$T_{i \alpha}=N_{i \alpha}-M_{i \alpha \beta, \beta}$,

with Greek indices ranging from 1 to 2 and Roman indices ranging from 1 to 3. For this work, we assume that graphene is reasonably well-approximated by assuming isotropy in plane. In this case,

$N_{i \beta}=A r_{i, \alpha}\left(v E_{\gamma \gamma} \delta_{\alpha \beta}+(1-v) E_{\alpha \beta}\right)-M_{i \eta \zeta} \Gamma_{\beta \eta \zeta}$

and

$M_{i \alpha \beta}=D n_{i}\left(v b_{\gamma \gamma} \delta_{\alpha \beta}+(1-v) b_{\alpha \beta}\right)$

where $r_{i, \alpha}$ are components of the surface metric tensor, $E_{\alpha \beta}$ are components of the Lagrange strain tensor, $\Gamma_{\beta \eta \zeta}$ are the Christoffel symbols, $\delta_{\alpha \beta}$ are components of the Kronecker delta, $n_{i}$ are components of the deformed surface unit normal vector, and $b_{\alpha \beta}$ are components of the surface curvature tensor. The $M_{i \alpha \beta}$ term represents the bending part of the stress, while the $N_{i \beta}$ term comprises both the membrane stretching part of the stress and an additional term $\left(M_{i \eta \zeta} \Gamma_{\beta \eta \zeta}\right)$ arising from the use of mixed bases.

Materials in a continuum shell are characterized by Poisson's ratio, $v$, in-plane rigidity, $A=$ $h E /\left(1-v^{2}\right)$, and flexural rigidity $D=h^{3} E /\left(12\left(1-v^{2}\right)\right)$, where $E$ is the Young's modulus and $h$ is the sheet thickness. Since thickness in graphene is not well-defined, we characterize the material by specifying effective values for $v, A$, and $D$ directly. In all of our simulations, we take $v=0.398$ and $A=288.74 \mathrm{~N} / \mathrm{m}^{11}$. The values chosen for the flexural stiffness, $D$, are discussed in the main text.

\section{Boundary Conditions}

For the annulus problem, we consider the outer and inner boundaries to be pinned. This corresponds to assigning position and specifying the applied couple per unit length, $c_{i}=M_{i \alpha \beta} v_{\alpha} v_{\beta}$ 
to be zero. The $v_{\alpha}$ are the components of the outward unit normal vector of the reference midplane edges. In the rectangular shear problem, the top and bottom edges are considered pinned, and are thus treated analogously to the annulus problem. On the initially vertical sides both the couple per unit length and the force per unit length,

$f_{i}=T_{i \alpha} v_{\alpha}-\left(M_{i \alpha \beta} v_{\alpha} \tau_{\beta}\right)_{, s^{\prime}}$

are set to zero. The $\tau_{\alpha}$ are the components of the unit tangent vectors of the reference midplane edges and the subscript $s$ denotes differentiation with respect to arc-length.

\section{Numerical implementation}

In order to use this plate theory to solve for equilibrium deformations, we embed Eq. (5) into a fictitious damped dynamical system,

$T_{i \alpha, \alpha}=\rho \ddot{r}_{i}+c \dot{r}_{i}$,

where $r_{i}$ are components of the position vector, $\rho$ is a mass parameter, and $c$ is a damping parameter. The long-time limit of this system is the equilibrium configuration of the sheet. This method, called dynamic relaxation, has long history of successful application to a variety of nonlinear elasticity problems involving sheets and cables ${ }^{12-15}$. The mass and damping parameters are set purely to ensure stability and enhance the rate of convergence.

To solve the system Eq. (S11) numerically, we require both spatial and temporal discretizations. To discretize the equations in space, we use Green's theorem to derive finite difference approximations of the required derivatives (see, e.g., ${ }^{13-15}$ ). In time, we use a basic central difference approximation,

$\dot{r}_{i}^{n}=\frac{1}{2}\left(\dot{r}_{i}^{n+\frac{1}{2}}+\dot{r}_{i}^{n-\frac{1}{2}}\right) ; \ddot{r}_{i}^{n}=\frac{1}{\Delta t}\left(\dot{r}_{i}^{n+\frac{1}{2}}-\dot{r}_{i}^{n-\frac{1}{2}}\right) ; \dot{r}_{i}^{n-\frac{1}{2}}=\frac{1}{\Delta t}\left(r_{i}^{n}-r_{i}^{n-1}\right)$,

where $n$ is the time step and $\Delta t$ is the time step size. In this work, we use a particular variant of dynamic relaxation called kinetic damping ${ }^{16,17}$. In this approach, the explicit damping term is set to zero and the kinetic energy of the entire system is tracked. When it reaches a peak, all of the nodal velocities are set to zero and the simulation is restarted. This can be more efficient than the standard viscous damping approach as it requires the regulation of two parameters (mass and time step size) instead of three.

In all our simulations, the timestep is set to 1 , and all simulations are run for 100000 steps as that led to configurations very close to equilibrium. The mass is varied manually in each simulation to maintain stability. For the annular problem, we use a mesh with 500 nodes circumferentially and 82 nodes radially distributed and logarithmically biased so that more nodes are located near the inner boundary. For the rectangular problem, we use a rectangular mesh of 150 by 150 nodes uniformly distributed. The in-plane initial conditions are set to match as best as possible those in the MD simulations and described in the previous section. In addition, we use a smooth initial out-of-plane displacement perturbation of the form,

$z / L=a \sin \left(\frac{k \pi x}{L}\right) \sin \left(\frac{k \pi y}{L}\right)$,

where $x, y$, and $z$ represent nodal coordinate positions and $\mathrm{L}$ is a non-dimensionalizing term. For the annulus problem, we set $L=9 \mathrm{~nm}, a=10^{-7}$, and $k=2$, while in the rectangle shear problem, we set $L=5 \mathrm{~nm}, a=10^{-7}$, and $k=1$. These values are chosen somewhat arbitrarily, but with 
the goal of facilitating convergence of the dynamic relaxation procedure.

\section{Computation of surface area}

The change in surface area of the deformed sheet is computed using an expression analogous to Nanson's formula ${ }^{15}$,

$\alpha n_{i}=\frac{1}{2} e_{i j k} e_{\alpha \beta} r_{i, \alpha} r_{j, \beta}$,

where $\alpha$ is the areal stretch, $r_{i, \alpha}$ are components of the surface metric tensor, and $e_{i j k}$ and $e_{\alpha \beta}$ are the components of the three- and two-dimensional unit alternator, respectively.

\section{$\underline{\text { References }}$}

(1) Plimpton, S. Journal of Computational Physics 1995, 117, 1-19.

(2) Stuart, S. J.; Tutein, A. B.; Harrison, J. A. Journal of Chemical Physics 2000, 112, 6472-6486.

(3) Brenner, D. W.; Shenderova, O. A.; Harrison, J. A.; Stuart, S. J.; Ni, B.; Sinnott, S. B. Journal of Physics-Condensed Matter 2002, 14, 783-802.

(4) Xu, Z. P.; Buehler, M. J. Acs Nano 2010, 4, 3869-3876.

(5) Cranford, S.; Sen, D.; Buehler, M. J. Applied Physics Letters 2009, 95.

(6) Qin, Z.; Feng, X. Q.; Zou, J.; Yin, Y. J.; Yu, S. W. Applied Physics Letters 2007, 91, 043108.

(7) Wei, Y. J.; Wu, J. T.; Yin, H. Q.; Shi, X. H.; Yang, R. G.; Dresselhaus, M. Nat. Mater. 2012, 11, 759-763.

(8) Lin, M.; Manocha, D.; Shewchuk, J., Triangle: Engineering a 2D quality mesh generator and Delaunay triangulator. In Applied Computational Geometry Towards Geometric Engineering, Springer Berlin Heidelberg: 1996; Vol. 1148, pp 203-222.

(9) Taylor, M.; Bertoldi, K.; Steigmann, D. J. J. Mech. Phys. Solids 2014, 62, 163-180.

(10) Arroyo, M.; Belytschko, T. International Journal for Numerical Methods in Engineering 2004, 59, 419-456.

(11) Lu, Q.; Huang, R. International Journal of Applied Mechanics 2009, 1, 443-467.

(12) Silling, S. A. Computer Methods in Applied Mechanics and Engineering 1988, 70, 251-273.

(13) Silling, S. A. J. Mech. Phys. Solids 1989, 37, 293-316.

(14)Haseganu, E. M.; Steigmann, D. J. Computational Mechanics 1994, 14, 596-614.

(15) Taylor, M.; Steigmann, D. J. Journal of Thermal Stresses 2009, 32, 448-476.

(16) Rezaiee-Pajand, M.; Kadkhodayan, M.; Alamatian, J.; Zhang, L. C. Computers \& Structures 2011, 89, 783-794.

(17) Topping, B. H. V.; Khan, A. I. Engineering Computations 1994, 11, 513 - 548. 
Table S1. Numerical values of the parameters entering in Eq. (S2). These values are obtained by fitting Eq. (S2) to the MD results.

\begin{tabular}{|c|c|c|c|c|c|c|c|}
\hline $\boldsymbol{R}_{o} / \boldsymbol{R}_{i}$ & $\boldsymbol{R}_{\boldsymbol{i}}(\AA)$ & A & C & $\exp (A)-C$ & B & $H_{\max }(\AA)$ & $N$ \\
\hline 2 & 22.5 & 2.545 & 12.41 & 0.33 & 0.028 & $\begin{array}{c}0.21 \Delta \theta-0.23 \\
2^{\circ}<\Delta \theta<10^{\circ}\end{array}$ & $9.3 \pm 1.2$ \\
\hline \multirow[t]{6}{*}{3} & 7.5 & 1.93 & 6.59 & 0.30 & 0.046 & $\begin{array}{c}0.2 d \theta-1 \\
5^{\circ}<d \theta<12^{\circ}\end{array}$ & 5 \\
\hline & 15 & 1.63 & 4.90 & 0.20 & 0.068 & $\begin{array}{c}0.11 \Delta \theta+0.36 \\
4^{\circ}<\Delta \theta<10^{\circ}\end{array}$ & $6.5 \pm 0.5$ \\
\hline & 22.5 & -0.095 & 0.74 & 0.17 & 0.31 & $\begin{array}{c}0.17 \Delta \theta-0.17 \\
2^{\circ}<\Delta \theta<10^{\circ}\end{array}$ & $8 \pm 1$ \\
\hline & 30 & -0.68 & 0.39 & 0.12 & 0.47 & $\begin{array}{l}0.21 \Delta \theta-0.1 \\
2^{\circ}<\Delta \theta<10^{\circ}\end{array}$ & $10.5 \pm 0.5$ \\
\hline & 37.5 & 0.35 & 1.37 & 0.05 & 0.26 & $\begin{array}{c}0.23 \Delta \theta, \\
2^{\circ}<\Delta \theta<10^{\circ}\end{array}$ & $11.5 \pm 0.5$ \\
\hline & 45 & -0.62 & 0.45 & 0.09 & 0.44 & $\begin{array}{c}0.35 \Delta \theta-0.38 \\
2^{\circ}<\Delta \theta<8^{\circ}\end{array}$ & $12 \pm 1$ \\
\hline 4 & 22.5 & 2.52 & 12.35 & 0.0 & 0.038 & $\begin{array}{c}0.26 \Delta \theta-0.54 \\
2^{\circ}<\Delta \theta<8^{\circ}\end{array}$ & $8.3 \pm 1.2$ \\
\hline 5 & 22.5 & 2.59 & 13.44 & -0.1 & 0.036 & $\begin{array}{c}0.30 \Delta \theta-0.75 \\
3^{\circ}<\Delta \theta<8^{\circ}\end{array}$ & $8.6 \pm 0.6$ \\
\hline
\end{tabular}

